\title{
Designing and Performance Evaluation of an Advanced Method for Corner Detection using Harris Technique
}

\author{
Amandeep \\ M. Tech, (ECE) \\ P.I.E.T, Smalkha (K.u.k) India
}

\author{
Parveen Singla \\ Electronics \& Comm. Engg. \\ P.I.E.T, Smalkha (K.u.k) India
}

\begin{abstract}
Corner detection is a significant task in different machine vision and image processing systems because corners play an important role in describing object unique features for recognition and identification. Corner detection comes within reach of computer visualization systems to extract certain kinds of characteristics and understand the contents of an image. In this work modify the Harris technique in order to enhance the performance of corner detection. To include the effect of Morphology technique on the modified Harris corner technique. Observe the simulation results of proposed detection technique \& compare results with traditional method. MATLAB R2013a has been used as an implementation platform using generalized MATLAB toolbox and image processing tool box.
\end{abstract}

\section{Keywords}

Harris Technique, Morphology Technique MATLAB etc.

\section{INTRODUCTION}

Corner detection is a critical task in various machine vision and image processing systems because corners play an important role in describing object unique features for recognition and identification. The initial operator concept of "points of interest" in an image, which could be used to locate matching regions in different images, was developed by Hans P. Moravec in 1977. The Moravec operator is considered to be a corner detector because it defines interest points as points where there are large intensity variations in all directions. Chris Harris and Mike Stephens in 1988 improved upon Moravec corner detector by taking into account the differential of the corner score with respect to direction directly, instead of using shifted patches [1]. Moreover, SUSAN and Harris detection algorithms were improved by selecting an adaptive gray difference threshold and by changing directional differentials. Without losing image data information, extracting corners can minimize the processing data. Therefore, corner detection has practical value and it plays an important role in scale space theory, motion tracking , image matching, building 2D mosaics, stereo vision, preprocessing phase of outline capturing systems, image representation and other fields [2]. Harris Corner Detector is proposed as a corner detection technique to extract palm print features in the form of corners. Hamming distance similarity measurement using sliding window method is used as a feature matching method for the corners detected. The aim of using hamming distance method for corner matching is the non-dependency of the method with the number of corners detected. So, the comparison (matching) time will be constant with hamming distance feature matching method. We used the same feature matching technique in edge detection and got good results [3].Three such modified techniques using Harris, Canny- Harris and Laplace-Harris with Harris operator on the basis of distances computed by these methods from user detected corners. Harris detection of corners in terms of preprocessing and hence reducing data has increased accuracy in terms of less distance detection from user detected corner points [4]. Bayes Shrink Technique proposed by Chang Yu and Vetterli, is an adaptive data-driven threshold for image de-noising via wavelet soft-thresholding. Generalized Gaussian distribution (GGD) for the wavelet coefficients is assumed in each detail sub band. It is then tried to estimate the threshold $\mathrm{T}$ which minimizes the Bayesian Risk, which gives the name Bayes Shrink [5]. The corners extracted by the three and the original scale spaces also had scale invariant property. The experiments results proved that, compared with the scale space on the whole Gaussian pyramid, the utilization factor of the image was increased, the calculation time is decreased, and the image was high recurrence rate and stability [5]. From the above it can be concluded that existing methods for corner detection retails some disadvantages and problems regarding their accuracy, time consumption for computation, efficiency. There is a need to propose an advanced method for the same, so as to minimize above said problems and disadvantages. A single threshold value is not suitable for general situation. The corner detector needs an improved adaptive threshold and the shape of mask can also be improved. Some of the existing methods are not isotropic (as pointed by Moravec) i.e. if an edge is present that is not in the direction of the neighbors (horizontal, vertical, or diagonal), then the smallest SSD will be large and the edge will be incorrectly chosen as an interest point. The disadvantage with some of the existing methods is it that they are not invariant to large scale changes in the image. Many techniques can only respond to 1 pixel wide lines at certain angles, when the quantization of the circle misses the line. Now we use convolution method, Gaussian filter and threshold value to Extraction of local maxima by performing a grey scale morphological. Dilation and then finding points in the corner strength image that match the dilated image and are also greater than the threshold. This paper is organized as follows. In Section 2, we propose Types of corner detection. In section 3 represent problem formulation. In Section 4 discuss about proposed method to modified Harris corner detection. Design examples and coefficient quantization effects are provided. A final result discussed in Section5 and conclusion discussed in section 6. In essence, we ask you to make your paper look exactly like this document. The easiest way to do this is simply to download the template, and replace the content with your own material.

\section{CORNER DETECTION METHOD}

1. ALL SUSAN (SMALLEST UNIVALUE SEGMENT ASSIMILATING NUCLEUS) CORNER DETECTORS

2. CURVATURE SCALE SPACE (CSS)

3. MORAVEC CORNER DETECTION ALGORITHM 


\section{HARRIS CORNER DETECTOR}

\subsection{SUSAN (SMALLEST UNIVALUE SEGMENT ASSIMILATING NUCLEUS) CORNER DETECTORS} [5]

This detector does not use spatial derivatives not smoothes the image. Instead, a circular mask is applied around every pixel, and the grey scale values of all the pixels within the mask are compared to that of the centre pixel (the "nucleus"). Calculate the number of pixels within the circular mask which have similar brightness to the nucleus [4].

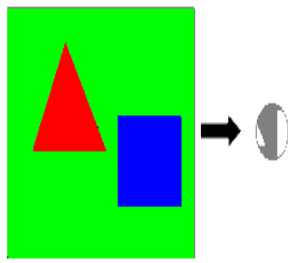

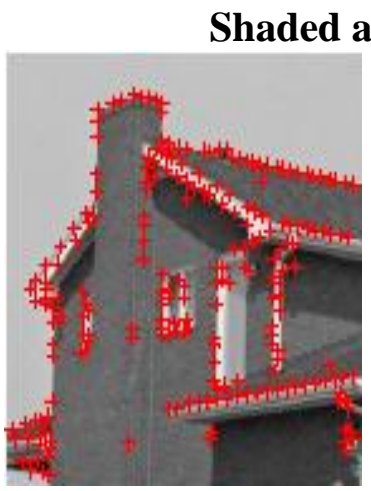

House.jpg

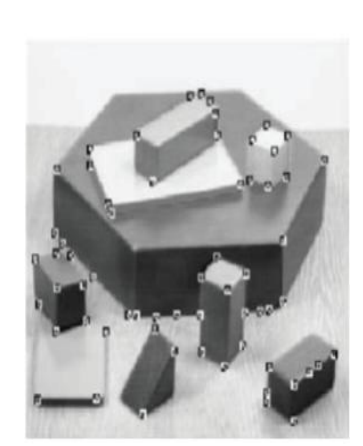

Block.jpg
Figure 1: Corner detection on the house.jpg and block.jpg using SUSAN technique.

Drawback

- A fixed global threshold is not suitable for general situation. The corner detector needs an improved adaptive threshold and the shape of mask can be improved, too

- The anti-noise ability is weak and the robustness of the algorithm should be strengthened.

\subsection{CURVATURE SCALE SPACE (CSS)}

The Curvature Scale Space (CSS) corner detector is very robust with respect to image noise, and is believed to perform better than existing corner detectors. The corner points are defined as the maxima of absolute curvature that are above a threshold value. Track the corners through multiple lower scales to improve localization. Compare T-corners to the corners found using the CSS procedure and remove very close corners. The CSS detector has been carried out for both edge detection and corner detection explicitly. The Curvature Scale Space (CSS) operator detects corners by directly looking for local maxima of absolute curvature.

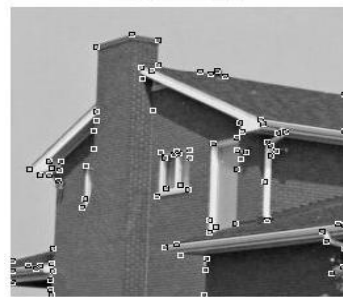

House.jpg

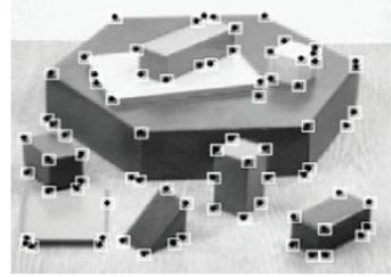

Block.jpg
Figure 2: Corner detection on the house.jpg and block.jpg using CSS technique.

Drawback

The obvious drawback in CSS is difficult to find corners in convex objects. Convex objects cannot be represented due to missing inflection points.

\subsection{The Moravec Corner Detection Algorithm}

The input of Moravec algorithm is gray-level image and the output is an image in which values are proportional to the likelihood that the pixels are corners. The similarity is measured by taking the sum of squared differences (SSD) between the two patches.

- A lower number indicates more similarity. If the pixel is in a region of uniform intensity, and then the nearby patches will look similar.

- If the pixel is on an edge, then nearby patches in a direction perpendicular to the edge will look quite different, but nearby patches in a direction parallel to the edge will result only in a small change.

- If the pixel is on a feature with variation in all directions. Then none of the nearby patches will look similar the corner strength is defined as the smallest SSD between the patch and its neighbors (horizontal, vertical and on the two diagonals). If this number is locally maximal, then a feature of interest is present.

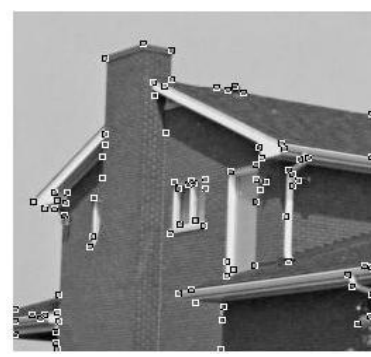

House.jpg

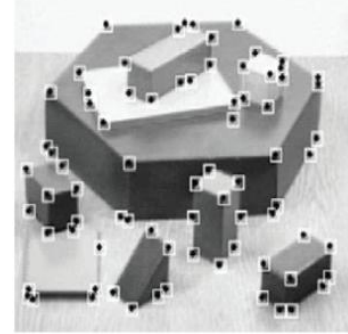

Block.jpg

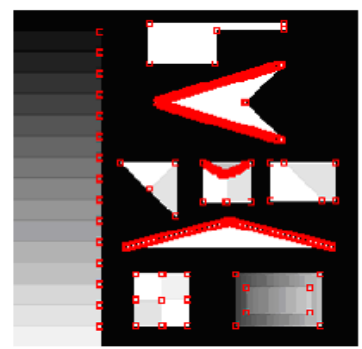

Article.jpg

Figure 3: Corner detection on image using Moravec algorithm 
Drawback in Moravec

- Noisy response due to a binary window function

- Only a set of shifts at every 45 degree is considered

- Responds too strong for edges because only minimum of $\mathrm{E}$ is taken into account

\subsection{Harris Detection Method [1]}

Harris corner detector is based on the auto correlation function of the signal. The basic idea of this detector is we find whether point shows significant change in all direction or not. If yes then point is marked as a corner point it requires larger corner response function to detect corner. Take the points of local maxima of $R$

Measure of corner response:

$$
R=\operatorname{det}(M)-k(\text { Trace } M) \wedge 2
$$

Where $\operatorname{det}(\mathrm{M})=\mathrm{AB}-\mathrm{C}^{\wedge} 2$

Trace $\mathrm{M}=\mathrm{A}+\mathrm{B}$

$\mathrm{k}=$ empirical constant, $\mathrm{k}=(0.04-0.06)$

$\mathrm{R}$ is positive for corners, negative for edges, and small for flat regions.

One can avoid setting the parameter $\mathrm{k}$ by using corner measure which amounts to the harmonic mean of the Eigen values

$$
M_{c}^{\prime}=2 \frac{\operatorname{det}(A)}{\operatorname{trace}(A)+\epsilon}
$$

Where $\epsilon=$ small positive constant.

\subsection{Morphological Processing}

A morphological operation on a binary image creates a new binary image in which the pixel has a non-zero value only. The structuring element is a small binary image, i.e. a small matrix of pixels, each with a value of zero or one. The matrix dimensions specify the size of the structuring element. The pattern of ones and zeros specifies the shape of the structuring element.

\section{TYPE OF MORPHOLOGICAL}

\section{- EROSION}

- DIALATION

\section{EROSION}

The basic effect of the operator on a binary image is to erode away the boundaries of regions of foreground pixels (i.e. white pixels, typically). Thus areas of foreground pixels shrink in size, and holes within those areas become larger
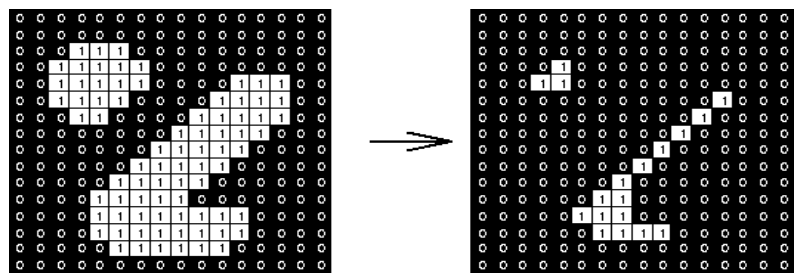

Figure 4: Effect of erosion using a $3 \times 3$ square structuringelement strip away a layer of pixels from an object, shrinking it in the process.

\section{DILATION}

The basic effect of the operator on a binary image is to gradually enlarge the boundaries of regions of foreground pixels (i.e. white pixels, typically). Thus areas of foreground pixels grow in size while holes within those regions become smaller.
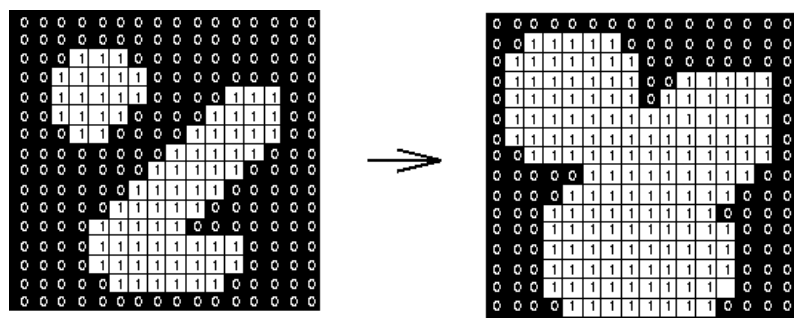

Figure 5: Effect of dilation using a $3 \times 3$ square structuring element strip away a layer of pixels from an object, shrinking it in the process

\section{PROBLEM FORMULATION}

From the above theory it can be concluded that existing methods for corner detection retails some disadvantages and problems regarding their accuracy, time consumption for computation, efficiency. Some of these problems are mentioned below:

- A universal problem with all the existing method is still an open problem as no good corner detector exists in terms of accuracy.

- $\quad$ Some existing methods wrongly assimilated the objects as corners when small image structures (including impulsive noise and thin lines) are eliminated.

\section{PROPOSED METHOD}

In this work we make some experiment on equation (1) and (2) But results are not getting accurate as ground truth in base paper. To increase the corner detection accuracy and efficiency of image as used in base paper in proposed method modify above formula and get the positive response with high accuracy. When second equation is dividing by 2 and gets equation as show below.

$$
M_{c}^{\prime}=\frac{\operatorname{det}(A)}{\operatorname{trace}(A)+\epsilon}
$$

Now we apply dilation morphology method on third equation and compare result with threshold value .Find the maxima value after match the corner image and dilation image which give accurate result.

\subsection{Steps For Proposed Corner Detection}

1. Inputting of test image.(.jpg., tiff, png, gif)

2. Conversion of $3 \mathrm{D}$ matrix of input image into $2 \mathrm{D}$ matrix.

3. Initialization of input parameters.

\footnotetext{
a. Standard deviation of smoothing Gaussian. $\quad \operatorname{sigma}(\sigma)=\mathbf{2 . 1}$

b. Threshold value for Harris response. corner threshold $=\mathbf{1 0 0 0}$

c. Radius of region considered in nonmaximal suppression. radius $\mathbf{=} \mathbf{3}$
} 
4. Computation of Image derivatives for $\mathrm{x}$ direction using $2 \mathrm{D}$ convolution method.

$$
\mathrm{X}=\mathrm{I} \otimes(-1,0,1) \sim d l / d x
$$

5. Computation of Image derivatives for $\mathrm{y}$ direction using $2 \mathrm{D}$ convolution method.

$$
\mathrm{Y}=\mathrm{I} \otimes(-1,0,1) \mathrm{T} \sim d l / d y
$$

6. Generation of Gaussian filter of size $6 *$ sigma $(+/-$ 3 sigma) and of minimum size $1 \times 1$.

7. Computation of Smoothed squared image derivatives in $\mathrm{x}$ direction using $2 \mathrm{D}$ convolution method. $\quad \mathbf{A}=\mathbf{X}^{2} \otimes \mathbf{w}$

8. Computation of Smoothed squared image derivatives in $\mathrm{y}$ direction using $2 \mathrm{D}$ convolution method. $\mathbf{B}=\mathbf{Y}^{2} \otimes \mathbf{w}$

9. Computation of Smoothed squared image derivatives in $\mathrm{x}$ and $\mathrm{y}$ direction using $2 \mathrm{D}$ convolution method. $\mathbf{C}=(\mathbf{X Y}) \otimes \mathbf{w}$

10. Corner Image $=\left(\mathbf{A B}-\mathbf{C}^{2}\right) /(\mathbf{A}+\mathbf{B})$

11. Applied morphological operation i.e. dilation on roughly corner image and then compare dilated image with corner image.

12. These steps greatly increase the accuracy of corner detection. Comparison of corner image and dilated image works jointly with comparison of corner image and threshold value.

\section{MATLAB CODE}

clc

close all

clear all

\%inputting of test image

input_image=imread('artical.jpg');

figure(1)

$\%$ display of test image

imshow(input_image)

title('original test image')

START=cputime;

$\%$ conversion of 3D matrix of input image into 2D matrix

if length(size(input_image)) $>2$

input_image =rgb2gray(input_image);

end

$\%$ initiallization of input parameters

sigma $=2.1 ; \%$ standard deviation of smoothing Gaussian

corner_threshold=1000; \% threshold value for Harris response or Harris corner threshold

radius $=3$; \% radius of region considered in non-maximal suppression

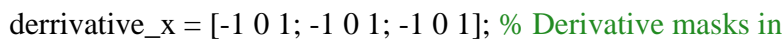
$\mathrm{x}$ direction

derrivative_y= derrivative_x';\% Derivative masks in y

direction

image_derrivative_x $=$ conv2(input_image, derrivative_x, 'same'); \% computation of Image derivatives for $\mathrm{x}$ direction using 2D convolution method..'same' returns the same size of output as that of image

figure(2)

imshow(image_derrivative_x)

title('x direction derrivative image') image_derrivative_y = conv2(input_image, derrivative_y, 'same'); \% Image derivatives for y direction using 2D

convolution method

figure(3)

imshow(image_derrivative_y)

title('y direction derrivative image')

$\%$ Generation of Gaussian filter of size $6 *$ sigma (+/3 sigma) and of minimum size $1 \times 1$.

\%this function returns a rotationally symmetric Gaussian lowpass filter of size specified with standard deviation sigma (positive).

$\mathrm{g}=$ fspecial('gaussian',max $\left(1, \mathrm{fix}\left(6^{*}\right.\right.$ sigma $\left.)\right)$, sigma $) ;$

$\%$ application of Gaussian filter on both image derrivatives and getting

$\%$ of Smoothed squared image derivatives

smooth_x_image $=$ conv2(image_derrivative_x. ${ }^{\wedge} 2, \mathrm{~g}$, 'same'); \% computaion of Smoothed squared image derivatives in $\mathrm{x}$ direction using 2D convolution method

smooth_y_image $=$ conv2 $\left(\right.$ image_derrivative_y. ${ }^{\wedge} 2, \mathrm{~g}$, 'same'); \% computation of Smoothed squared image derivatives in y direction using $2 \mathrm{D}$ convolution method smooth_xy_image $=$ conv2(image_derrivative_x.*image_derrivative_y, g, 'same'); $\%$ computation of Smoothed squared image derivatives in $\mathrm{x}$ and $\mathrm{y}$ direction using $2 \mathrm{D}$ convolution method

corner_image $=\left(\right.$ smooth_x_image.* ${ }^{*}$ smooth_y_image smooth xy image.^2)./(smooth_x_image + smooth_y image + eps); \% measurement of corner using smoothed squared image derrivatives in $\mathrm{x}, \mathrm{y}$ and $\mathrm{xy}$ direction

figure(4)

imshow(corner_image)

title('corner image')

$\%$ Extraction of local maxima by performing a grey scale morphological

$\%$ dilation and then finding points in the corner strength image that

$\%$ match the dilated image and are also greater than the threshold.

size_mask $=1 *$ radius $+1 ; \quad \%$ computation of Size of mask to be used in 2-D order-statistic filtering of corner strength image

dilated_image =

ordfilt2(corner_image,size_mask ${ }^{\wedge} 2$,ones(size_mask)); \% 2-D order-statistic filtering or Grey-scale dilation of corner

\%strength image using mask.

figure(5)

imshow(dilated_image)

title('dilated corner image')

corner_image $=$

(corner_image $==$ dilated_image) $\&($ corner_image $>$ corner_thre shold);\% Finding of maxima or finding points in the

\%corner strength image that match the dilated image and are also greater than the threshold.

figure(6)

imshow(corner_image)

title('threshold dilated corner image')

\section{STOP=cputime-START}

$[\mathrm{r}, \mathrm{c}]=$ find (corner_image);

$\%$ Finding of row,col coordinates for non zero elements in new maxima binary image. 
corner_detected=length $(\mathrm{c})$

figure(7)

imagesc(input_image)

axis image

colormap(gray)

hold on

plot(c,r,'bs'),

title('corners detected on original test image');

figure(8)

subplot(121)

imshow(input_image)

title('original test image')

subplot(122)

imagesc(input_image)

axis image

colormap(gray)

hold on

plot(c,r,'bs'),

title('corners detected on original test image');

$\% \% \%$ counting of corners

total_corners=bwarea $($ corner_image $)$

\section{RESULT}

An algorithm for corner detection using advanced Harris corner detection is proposed in this work. For experimental setup we have three images i.e. "house.bmp", "block.jpg" and "artical.jpg". Parameters i.e. number of corners detected have been taken for performance evaluation of proposed method.

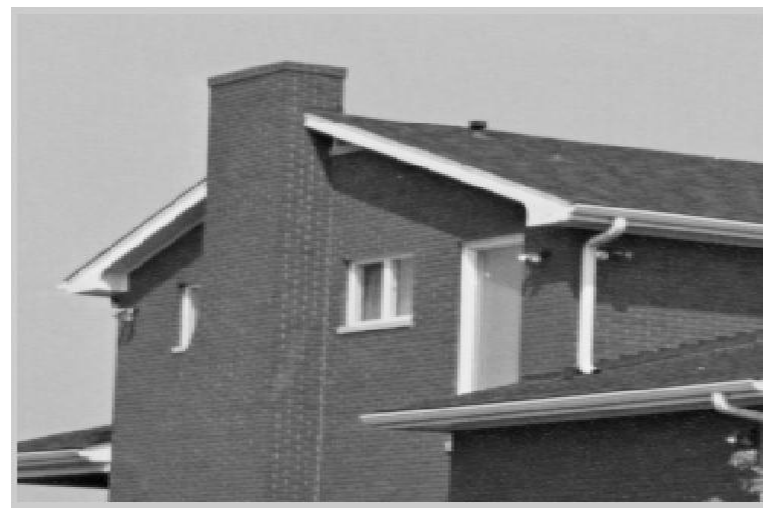

Figure 6: House.jpg test image

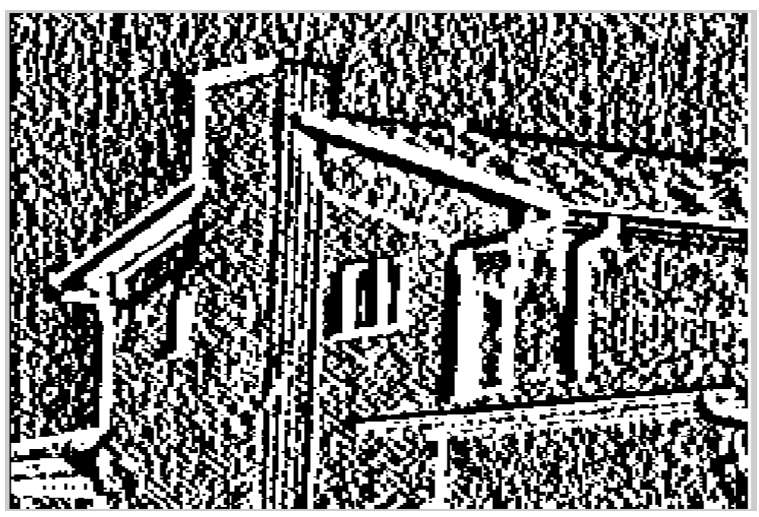

Figure 7: Horizontal gradient

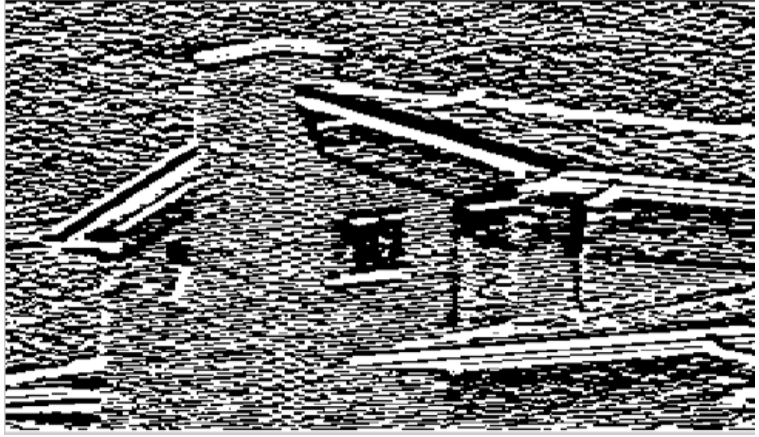

Figure 8: Vertical gradient

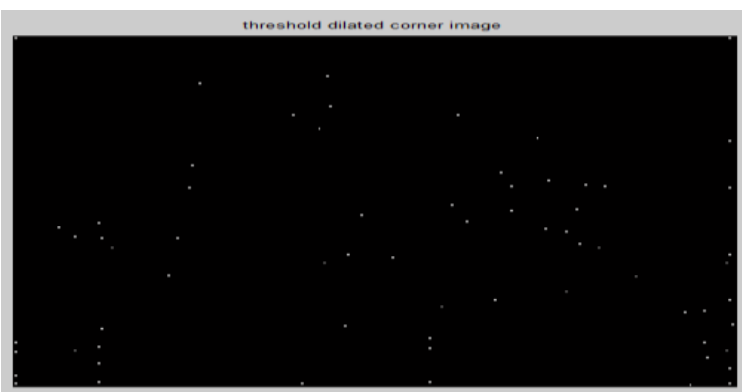

Figure 9: Threshold dilated image

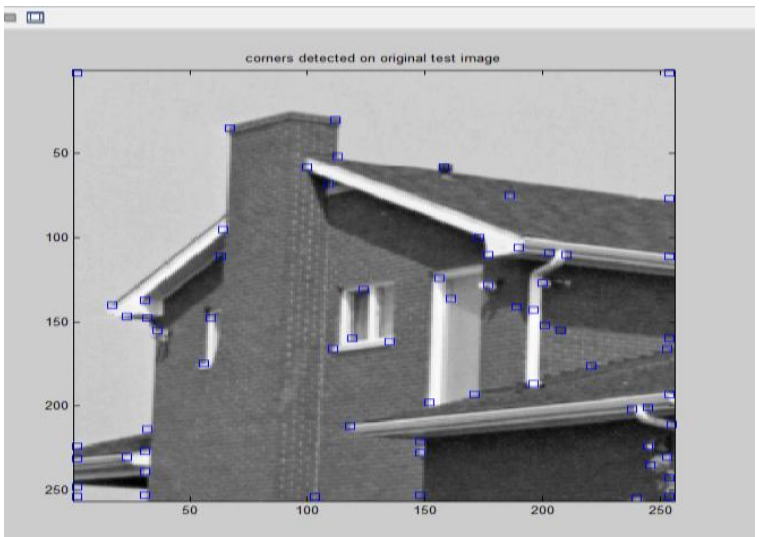

Figure 10: Corners on test image

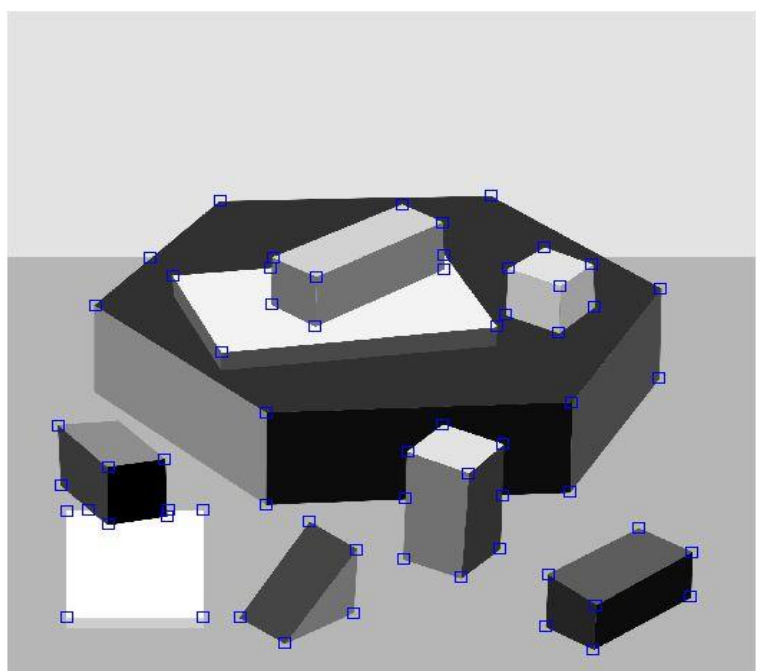

Figure 11: Final results obtained from applying proposed method on "block.jpg" 


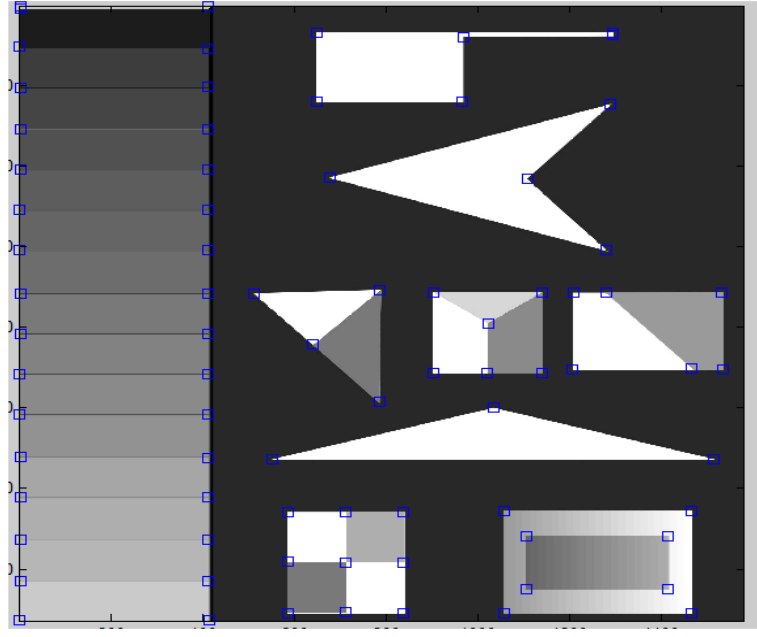

Figure 12: Final results obtained from applying proposed method on "artical.jpg"

\subsection{Comparison Of Performance Of Proposed Method With Existing Methods}

Table 1

\begin{tabular}{|c|c|c|c|}
\hline \multirow{2}{*}{$\begin{array}{l}\text { CORNER } \\
\text { DETECTION } \\
\text { METHOD }\end{array}$} & \multicolumn{3}{|c|}{$\begin{array}{l}\text { NUMBER OF CORNER IN TEST } \\
\text { IMAGE }\end{array}$} \\
\hline & $\begin{array}{l}\text { Block.j } \\
\text { pg }\end{array}$ & House.jpg & Article.jpg \\
\hline Ground truth[4] & 64 & 77 & 78 \\
\hline Harris[4] & 52 & 57 & 78 \\
\hline SUSAN[4] & 48 & 62 & 78 \\
\hline $\mathrm{CSS}[4]$ & 55 & 50 & 78 \\
\hline INLST[4] & 55 & 57 & 132 \\
\hline MSBT[4] & 57 & 64 & 78 \\
\hline Proposed & 62 & 75 & 78 \\
\hline
\end{tabular}

Performance evaluation parameters i.e. number of corners detected of Three Image (Block.jpg, House.jpg and Artical.jpg) have been given in table 1. Which give information for Corner detection for Block image by proposed method are 61 and that by ground truth are 64 , which is much closer as compared to other existing methods. In house image corners detected by proposed methods are 75 and that by ground truth are 77 , which is also much closer as compared to other existing methods. Same in the case of Article image corners detected by proposed methods are 79 and that by ground truth are 78 , which is also much closer as compared to other existing methods. So, propose method working efficiently as compared to other method. Experimental results on some standard test images show the effectiveness of the proposed corner detector in terms of both detection rate and localization accuracy.

\section{CONCLUSION}

The proposed method works on the basis of advanced technique for finding the local maxima or non-maximal suppression, which enhances its efficiency in terms of finding accurate corners. Moreover, in order to remove the trivial corner-like structures. Proposed result proof the above statement. Proposed method is working efficiently on all the test images.

\section{REFERENCES}

[1] Harris, C., Stephens, M.: A combined corner and edge detector. Proc. 4th Alvey Vision Conference, pp. 147151 (1988)

[2] Han Wang" Gray Level Corner Detection" IAPR Workshop on Machine Vision Applications. Nov. 17-19. 1998, Makuhari, Chiba. Japan

[3] Akshay Bhatia" Hessian-Laplace Feature Detector and Haar Descriptor for Image Matching" Ottawa, Canada, 2007

[4] Lin Zhang" A Multi-Scale Bilateral Structure Tensor Based Corner Detector" Biometrics Research Center, Department of Computing The Hong Kong Polytechnic University Hong Kong, China

[5] Smith, Stephen M. and J. Michael Brady, "SUSAN -- A New Approach to Low Level Image Processing", International Journal of Computer Vision, 1997. 